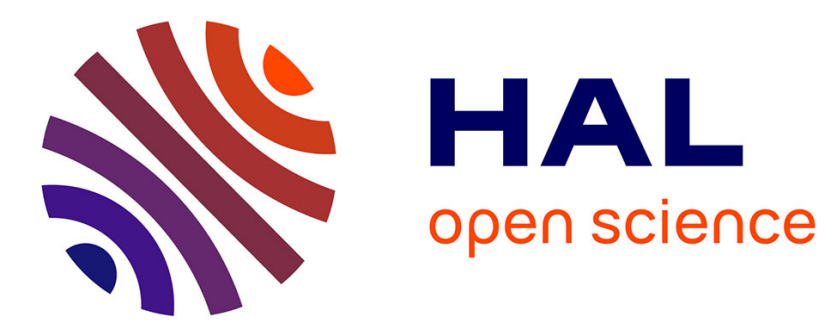

\title{
Real space small-angle scattering device
}

\author{
V. Lebedev, A. Dudakov, L. Cser, L. Rosta, Gy. Török
}

\section{To cite this version:}

V. Lebedev, A. Dudakov, L. Cser, L. Rosta, Gy. Török. Real space small-angle scattering device. Journal de Physique IV Proceedings, 1993, 03 (C8), pp.C8-481-C8-485. 10.1051/jp4:19938100 . jpa00252230

\section{HAL Id: jpa-00252230 https://hal.science/jpa-00252230}

Submitted on 1 Jan 1993

HAL is a multi-disciplinary open access archive for the deposit and dissemination of scientific research documents, whether they are published or not. The documents may come from teaching and research institutions in France or abroad, or from public or private research centers.
L'archive ouverte pluridisciplinaire HAL, est destinée au dépôt et à la diffusion de documents scientifiques de niveau recherche, publiés ou non, émanant des établissements d'enseignement et de recherche français ou étrangers, des laboratoires publics ou privés. 


\title{
Real space small-angle scattering device
}

\author{
V.T. LEBEDEV, A.D. DUDAKOV, L. CSER* ${ }^{*}$ L. ROSTA* and Gy. TÖRÖK* \\ PNPI, 188350 Gatchina St. Petersburg distr., Russia \\ * Research Institute for Solid State Physics, P.O. Box 49, 1525 Budapest, Hungary
}

\section{THE BASIC IDEA}

It is well known that during the course of any small-angle scattering experiment the differential cross-section - $d \sigma / d \Omega(\vec{q})$ is determined, where $\vec{q}$ - the momentum transfer vector - is given by the difference of the momenta of the incoming and scattered neutrons, $\overrightarrow{k_{0}}$ and $\vec{k}$,' respectively. The resolution power of a conventional experiment depends on the accuracy of the momentum values of the neutrons under consideration, any increase in the resolution gives rise to a decrease in the intensity of the neutron beams. In reality one needs only precise knowledge of the value of the momentum transfer independently of the the accuracy of $\vec{k}^{\prime}$ and $\overrightarrow{k_{0}}$. Then, there are no rigorous restrictions imposed on the collimation of the beams, i.e. the divergency of the neutron beams can be much wider, thus the intensity is increased appreciably. In this aspect the method under consideration is analogous with the neutron spin echo principle, which is based on measurement of the energy change of the scattered neutrons instead of determination of their initial and final energy separately. The method proposed below allows one to increase the luminosity of the performance via focused beams for investigating small particles, or to use a diverging beam for studying of large particles.

\section{SOME MATHEMATICAL CONSIDERATIONS}

The basic idea of the above approach can be seen from Fig.1. A grid installed in the beam produces a periodic modulation of the ingoing beam of initial flux distribution $\Phi(\vec{r})$. A second grid (called analyser), between the sample and the detector, modulates the scattered beam. The functions describing the transmission of these two modulators are denoted by $T_{M}(\vec{m} . \vec{r}+\phi)$ and $T_{A}(\vec{m} . \vec{r}+\psi)$ respectively. Here the radius vector $\vec{r}$ identifies the points in the plane of the modulators, while vector $\vec{m}$ gives the length and direction of the modulation $(\Lambda)$ in the same planes, i.e. $\Lambda=2 \pi /|\vec{m}|$.

$\phi$ and $\psi$ describe the phases of the transmission functions. The shape of these two functions are shown in the Fig.2. Note that the period of modulation $(\Lambda)$ of both grids is equal, however, their shapes are different. The intensity distribution behind the second modulator without sample has the shape

$$
i_{A}(\vec{r}, \vec{m})=T_{M}(-\vec{m} \cdot \vec{r}-\phi) \cdot \Phi(-\vec{r}) \cdot T_{A}(\vec{m} \cdot \vec{r}+\Psi)
$$


The small-angle scattering process gives rise to a deviation of the scattered neutron from the initial direction. This deviation we characterize by a vector $\vec{\rho}$ with coordinates $\Delta x$ and $\Delta y$ at the plane of the analyser. The components of the momentum transfer are

$$
q_{x}=2 \pi \Delta x /(\lambda L) \equiv(2 \pi / \lambda) \Theta_{x}
$$

and

$$
q_{y}=2 \pi \Delta y /(\lambda L) \equiv(2 \pi / \lambda) \Theta_{y}
$$

where $\mathrm{L}$ is the distance between the sample and the modulators. The measured intensity then turns up as an integral over scattering angles

$$
\begin{gathered}
I(\vec{m}, \vec{\rho})=(1 / S) \int d \vec{r} \Phi(-\vec{r}) T_{M}(-\vec{m} \cdot \vec{r}-\phi) T_{A}\left(\vec{m} \cdot \vec{r}+\Psi+m_{x} \Delta x+m_{y} \Delta y\right) . \\
(d \rho / d \Omega)(\Delta x / L)(\Delta y / L)
\end{gathered}
$$

Here $S$ is the area of the beam cross section at the place of the sample. When implementing the above principle one has to satisfy two basic conditions: first, one has to avoid the access of the direct beam to the detector; second, The higher harmonics of the Fourier integrals type (3) have to be eliminated. The first condition can be satisfied by the appropriate choice of the relative phase of the two modulators. The second condition requires the special choice of the profile of the transmission functions of the modulators. As the first modulator has a rectangular profile the Fourier representation contains only odd components, whereas the second one - having a semi- sinusoidal shape (Fig. 2) except, for the first harmonics - possesses only even harmonics

$$
\begin{gathered}
T_{M}(x)=(1 / 2)+(2 / \pi) \cos (x)+m_{1} \cos (3 x)+\ldots \\
+m_{n} \cos ((2 n+1) x)+\ldots
\end{gathered}
$$

where

$$
\begin{gathered}
m_{n}=2(-1) /(\pi(2 n+1)) \\
T_{A}(x)=(1 / \pi)-(1 / 2) \cos (x)+a_{1} \cos (2 x)+\ldots a_{n} \cos (2 n x)
\end{gathered}
$$

where

$$
a_{n}=2(-1) / \pi(2 n-1)(2 n+1)
$$

Under these conditions integral (3) can be rewritten as

$$
I=\left(\int \Phi(\vec{r}) d \vec{r} /(2 p S)\right)\left[\int(d \sigma / d \Omega) d \Omega-\int(d \sigma / d \Omega) \cos (\vec{m} . \vec{\rho}) d \Omega\right]
$$


Normalizing to the density of the ingoing beam intensity

$$
I_{i}=(1 / S) \int \Phi(\vec{r}) d \vec{r}
$$

and introducing the variable

$$
\vec{R}=(\lambda L / \Lambda) \vec{m} /|\vec{m}|
$$

expression (6) can be transformed to

$$
I(\vec{R})=\left(\lambda^{2} / 2 \pi^{3}\right)\left[\int(d \sigma(\vec{q}) / d \Omega) d \vec{q}-\int(d \sigma(\vec{q}) / d \Omega) \cos (\vec{q} \cdot \vec{R}) d \vec{q}\right]
$$

The second term is the correlation function in the real space $g(\vec{R})$.

\section{PERFORMANCE AND TEST}

The ingoing monochromatized neutron beam $(\langle\lambda\rangle=6.5 \AA$ and $\Delta \lambda / \lambda=0.3)$ of $10 \mathrm{~mm}$ diameter with divergency of $\pm 20^{\prime}$ was shaped by Cd diaphragms and focused to the sample using a curved mirror coated with ${ }^{58} \mathrm{Ni}$. The first modulator consists of parallel strips made of $\mathrm{Al}$ and $\mathrm{Cd}$ with periodicity of $\Lambda=0.92 \mathrm{~mm}$. The thickness of the Cd strips was $0.5 \mathrm{~mm}$. At the front of the sample two perpendicularly oriented grids were installed which divided the beam area into small $-0.5 \star 0.5 \mathrm{~mm}^{2}$ - point like spots. The second modulator was also formed of stripes of $\mathrm{Al}$ and $\mathrm{Cd}$. In order to produce the sinusoidal modulation the thickness of the Cd layers obeyed the law

$$
d(x)=-(1 / \mu) \ln [\cos (2 \pi x / \Lambda)]
$$

where $\mu$ is the linear coefficient of absorption. The detector system was assembled of eight linear $\mathrm{He}$ detectors covering a solid angle range of $7.10^{-3}$ steradian. The distances between the modulators and the sample ( $L$ ) was kept identical and changed symmetrically between the limits of $2 \mathrm{~cm}$ up to $17 \mathrm{~cm}$. (These limits were imposed by the cramped conditions). The limitation in $L$ gave rise to limitation of $R_{x}$ and $R_{y}$ values (see formula (8)), which caused certain limitation in the resolution power. The measurement was carried out by recording the intensity distribution as a function of the value $L$, i.e. the separation of the modulators. It is clear, that at the given orientation of the modulators the scanning can be carried out only by one of the components of the vector $\vec{R}$. For the second - perpendicular - component the so called high-slit geometry conditions are valid. Thus, the full course of measurement will be performed in two steps (by scanning in the $x$ and afterwards by scanning in the $y$ directions). The data obtained have to be corrected for the high-slit geometry distortions. In order to test the validity of the proposed method two suspensions of spherical latex particles in water were used as samples. The diameter of the latex spheres was $710 \AA$ and $2500 \AA$ respectively. These sizes were independently measured by light scattering correlation method. 
The size distribution was shown to be about $30 \%$. The concentrations of the latex particles were $30 \mathrm{w} \%$ and $25 \mathrm{w} \%$ respectively. For calibration, pure water was used which is assumed to be a fully incoherent scatterer. The experiment was carried out without focusing geometry, i.e. in such a way which is advantageous for the measuring of large particles. The experimental results are shown in Fig. 3.

It is seen that the steeper curve belongs to the smaller particle size, while the less steep one belongs to the larger particles. Both curves can be well approximated by an exponential

$$
g(R)=\exp \left[-R^{2} /<r^{2}>\right]
$$

i.e. the Guinier approximation is valid. Estimation of the radii $\left(r_{1}\right.$ and $\left.r_{2}\right)$ of the particles in this approximation gives $r_{1}=610 \pm 100 \AA$ and $r_{2}=1800 \pm 100 \AA$. These values are fairly close to those determined by the light scattering method.

\section{FUTURE PROSPECTS}

There are at least two ways to develop of the method proposed above. First, from the eq. (8) it is seen that parameter $\vec{R}$ can be changed instead of varying the distances separating the sample and the modulators by the change of the wavelength. Thus, at pulsed source the time-of-flight method gives the $I(\vec{R})$ distribution at properly fixed position of the modulators. Second, the surface scattering requires only one-dimensional scanning, so the usefulness of this method is evident. Moreover, carrying out the surface scattering in the time-of-flight performance makes the method especially attractive. 


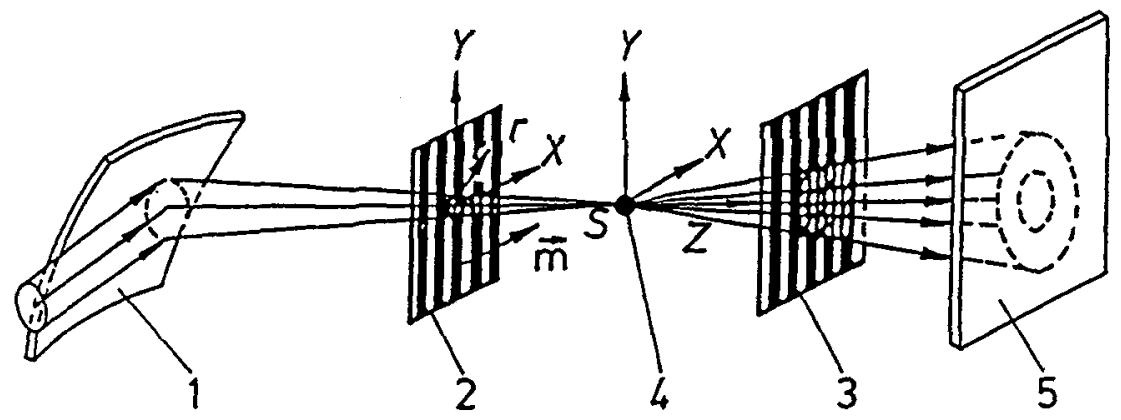

1.Schamatic layout of the rall spece smali-angle scuttering device. (Focusing version). 1. Focusing mirror; 2,3. modulators; 4. samplo; 5. detector.

$(x, y, z)$ defines the Descartes coordlate system; $\vec{r}$ is the vector determining the position of polnts in the plane belng parallel with the modulators, $\vec{m}$ lis the vec. tor of modulation (soo the text).

Fig.1.
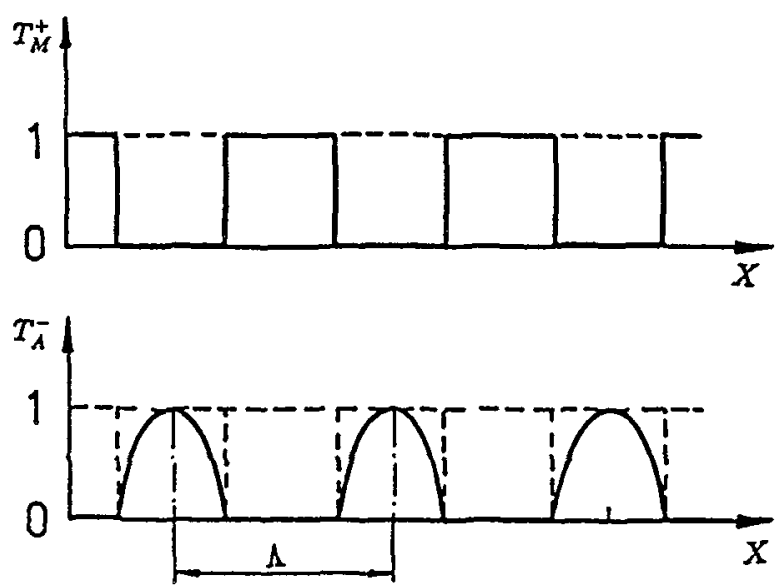

2. Transmiseion functlon of the two modulator. The upper eurve is the modulator transmission (No.3. In the Fig.I), the lower curve shows the tranimiselon of analyeer,- the socond modulator - (No.S. in the If. 1).

Fig.2.

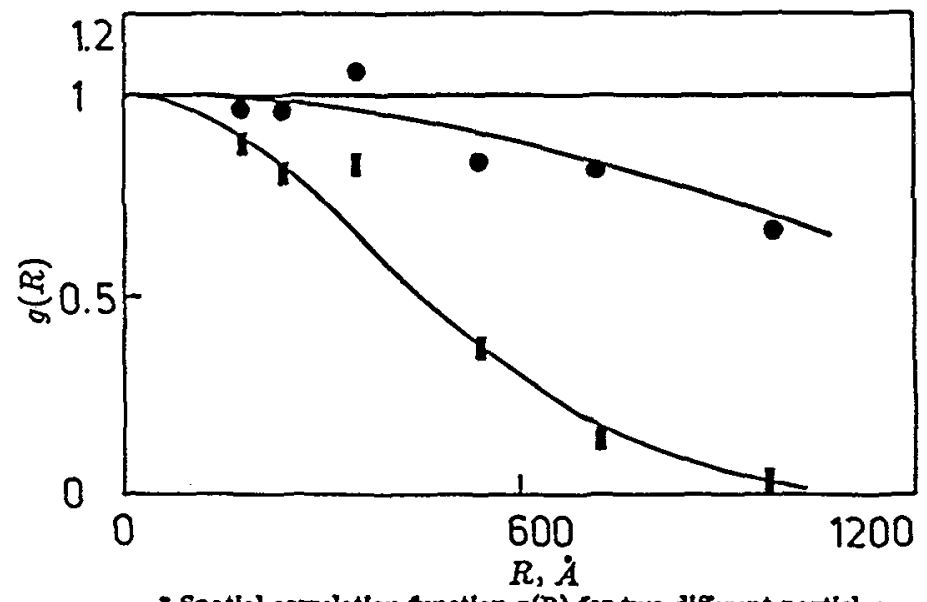

3.Spatial corralation truetion $g(R)$ for two different perticles. $1(0), d=710 \lambda, 2(\bullet) ;-d=2500 \lambda$;

Full lines calculation according the formule $\left(g(R)=\exp \left(-R^{2} /<r^{2}>1\right)\right.$

\$18.3. 